\title{
PCMusic - Support during Pain Management, Part 1
}

\author{
Ann-Sofie Paulander, , ${ }^{1,}$ Louise Eulau ${ }^{2}$ \\ ${ }^{1}$ Department of Music Education, Royal College of Music, Box 27711, SE-115 91 Stockholm, Sweden \\ ${ }^{2}$ Department of Nursing Science, Sophiahemmet University, P O Box 5605, SE-114 86 Stockholm, Sweden
}

Received February 7, 2020; Revised May 12, 2020; Accepted May 20, 2020

Copyright $(2020$ by authors, all rights reserved. Authors agree that this article remains permanently open access under the terms of the Creative Commons Attribution License 4.0 International License

\begin{abstract}
Although effective pain management interventions and programmes exist, provision of these services is regarded as inconsistent. There is evidence that multi-disciplinary methods are cost-effective in the treatment of pain, addressing the psychosocial, behavioral and biomedical aspects of pain states. It has been reported that music therapy and/or listening to music has a positive effect on the autonomic nervous system by reducing stress, focusing attention and effecting emotions, which in turn reduces pain experiences. The most effective way of pain reduction has been recorded while listening to selfselected music chosen for enjoyment or desired personal effect. This paper elaborates on phenomenologically describing an interdisciplinary method, Person-centered music (PCMusic), a complementary pain reducing treatment where music is a key component. A secondary goal is that the method can be used in other areas than pain management. The adopted concept integrates knowledge and methods from multiple disciplines viz. music therapy, caring science and Integrated Mental Training (IMT). PCMusic is discussed from a phenomenological perspective followed by a discussion of the music therapist role as a provider in care. The question of selfmanagement and the use of preferred or self-selected music in pain management are also addressed.
\end{abstract}

Keywords Pain Management, Music Therapy, Personcentered Care, Integrated Mental Training

\section{Introduction}

This is the first article of two presenting PCMusic, a method which can be used as a complementary treatment for pain management. This paper describes the theory and details of the method, and the second (paper) presents a mixed-method case study in where the method is clinically tested. The idea behind PCMusic originated when the authors exchanged experiences and knowledge concerning music therapy and caring science. Similarly to Olofsson and Fossum[1] who studied music therapy in cancer care, the authors of this article experienced differences in knowledge and performance between professionals in caring science and music therapy when music is used as a complementary treatment of pain. The authors recognized that a mutual interest in the use of music was present, but that there is a lack of procedures and concepts connecting music and caring in common theories.

\section{Pain}

The International Association for the Study of Pain defines pain as "An unpleasant sensory and emotional experience associated with actual or potential tissue damage, or described in terms of such damage" [2]. Neuroscientists have investigated acute pain meticulously, while chronic pain syndromes have not been researched to the same extent [3].

Melzack[3] describes pain as a complex process produced by patterns of nerve impulses generated by a widely distributed network. The network, "body-self neuromatrix", contain input factors as well as output factors. Input to the matrix are cognitive-evaluate, sensory-discriminative and motivational-affective factors. Output are pain perception, action programmes and stressregulation factors. Pain should therefore be seen and investigated as a multi-dimensional experience produced by multiple influences which result in holistic experiences of the body with different qualities at different times.

Melzack's theory implies that the cortex can modify and restrain pain signals by affecting and controlling inhibitory messages. Emotions are important since they are motivational-affective input factors that hereby can modify and restrain pain signals.

Both acute-and chronic pain management interventions and programmes do exist, though provision of these services is regarded as inconsistent. In particular, chronic pain is not receiving the appropriate priority considering its 
impact on individuals and society [4].

Researchers suggest that multi-disciplinary approaches are needed to address the multifaceted psychosocial, behavioral and biomedical aspects of pain, especially chronic pain, suggesting that intervention with selfmanagement strategies is one way of improving physical and psychological wellbeing $[5,6]$.

There is evidence that multi-disciplinary treatment methods are cost-effective for the treatment of acute and chronic pain [6].

\subsection{Music and Pain Management}

Listening to music is the primary way of using music in pain management. To obtain pain relief it is important that the music chosen is known or well known to the patient, a fact supported by research in neuropsychology [7]. The method of selecting the used music varies; i) self-selected music (the patient's own free choice of music), ii) preferred music (choice of music from a pre-set collection) or, iii) music chosen by staff.

Listening to music can arouse profound emotions in humans [7]. Music can affect the autonomic nervous system by reducing stress, focusing attention and effecting emotions in a positive way, which in turn, reduces pain experiences [8], as shown in e.g. the systematic review of 42 randomized controlled trials where the effects of music are shown during interventions in perioperative settings [9].

However, a necessary condition for music listening to have this positive effect is that the music in question evokes positive emotions [10].

The correlation between music and positive emotions when listening to self-selected music is known, since positive emotions correlate inversely with feelings of stress in everyday life [11].

The frequency of positive emotions is related to selfreported overall positive health in patients with pain since enhancing emotional engagement in music also enhances pain reduction and pain tolerance. The effect of positive emotions was shown in three experimental studies where the effects of using a cold pressor in combination with preferred music listening. The first study involved 80 participants, the second 72 participants and the third 55 participants [12].

Self-selected music and preferred music appear to stimulate feelings of control based on personal familiarities, memories and associations of the past, which in turn promotes a sense of control over pain [13]. In situations of acute pain and anxiety, it seems that the choice of music has to be music familiar to the patient i.e. of the patient's own choice. In situations of chronic pain and anxiety, it has been shown that a "New Age" type of music, such as Enya or natural soundscapes is effective [14].

Another important factor is listening habits. Today we allow all kind of music to be "pervasive" since we have a personal relationship to music [15]. Such a relationship consequently contains emotional and associative factors with significant personal meaning and memories [16].

Research concerning music selection, pain and music listening show ambiguities.

Preferred music selection demonstrates efficiency for relieving induced pain and increasing tolerance, compared to baseline [17].

In a study using self-selected music selection, 54 participants experiencing pain induced by cold pressure, showed a significantly higher pain tolerance and -control compared with participants listening to preferred relaxing music and sounds of white noise. Females rated pain intensity significantly lower while listening to preferred music than males [18]. The efficacy of self-selected music is attributed to evoking emotional responses and distraction of attention [19]. The effects of listening to pleasant or unpleasant music experiencing thermal skin pain was studied using eighteen healthy volunteers who were listening to pleasant or unpleasant musical excerpts matched to their level of arousal. In this study, only music experienced as pleasant produced significant pain reduction [20].

When twenty females responding to tonic heat pain, listened to self-selected music and pieces of music they perceived as pleasant, the result was a decrease in both pain and stress [21].

These results indicate that it is the valence of music, rather than the mood, which is the likely mediator of the hypoalgesic effect. One conclusion is therefore that for pain-management, self-selected music chosen for enjoyment or desired personal effect is more effective [22].

It is believed that there exists a difference between how stimulative and sedative music affects the biological system; the effects of stimulatory music are systematical and pronounced, whereas the effects of the sedative music are less predictable. This was for example shown in a study where 38 volunteers were asked to select favorite "stimulative" music and "sedative" music in a random order [23].

The majority of research results in the field of music and pain are based on experimental studies of induced pain, and it should be noted that induced pain is different from pain resulting from injuries or illness [22]. The effects of music listening in daily life in the context of pain and stress is also still understudied, as are the psycho-biological mechanisms that promote the health beneficial effects of music listening [24, 25].

Clinical results from acute pain interventions e.g. of forty-four orthopedic patients during post-operative bed rest indicated that patients listening to preferred music experienced a significant decrease of pain but, interestingly, no impact on their nervous system could be observed [26].

Further, parents singing with newborn infants may improve homeostasis during painful procedures although a statistically significant effect on the infants' pain score 
could not be observed [27].

The question of the need of physical presence of a music therapist during caring intervention procedures combining music and/or medical treatment has been stated. The research suggest that therapist presence is positive, it is recommended that music therapists are included in the caring teams [28]. The therapists add expertise and inclusion of their assessment offer emotional support.

A working model of how to use music therapy as an explicit procedural support in medical procedures is described where the music therapist engages in a reflexive process, which in turn, involves a continuous assessment of the patient's responses [29]. The purpose of the process is to be able to respond to, change, or refocus the intervention, for example: alter the music, focus of attention, or, any other part of the on-going interaction.

Combining these findings, we find that since pain can be seen as a perception-emotion rather than a physical concept, music listening and music therapy can be used for pain relief [8]. Music listening is regarded as a low-cost treatment available to many patients with pain [30] and self-selected music can quite easily be brought into different kinds of environments using e.g. mobile phones or mp3_players hereby offering control over pain relief and pain in a multitude of settings e.g. home, work, clinics, hospitals and care centers.

\section{Aim}

This paper describes the interdisciplinary method PCMusic, which is suggested as a complementary treatment for pain management within healthcare and medicine. PCMusic employs self-selected music as method of music selection. An important aspect of PCMusic is the aim of inspiring healthcare professionals to collaborate with music therapists when music is used in caring and medical contexts. Another goal is that the method can be used in other areas than pain management.

\section{PCMusic - Person-centered Music}

PCMusic is derived from a desire to integrate knowledge and methods from different disciplines viz. i) music therapy, ii) caring science and iii) Integrated Mental Training (IMT). The method is designed as a music experience used for treatment in healthcare, patient -assessment and evaluation. The philosophical framework for the method is the phenomenological life-world perspective as it offers a common denominator for the disciplines that are part of the method.

\subsection{Phenomenological Life-world Perspective}

Since the areas of music therapy, caring science and treatment of pain lack a common theory, we use the phenomenological life-world perspective $[31,32]$ as a theoretical framework for PCMusic. The perspective highlights how we live in the world as an integrated whole with relations to things and people. The perspective also fits well with theories of person-centered care that is intended for caring science as well as to the music therapy referred here.

The adopted perspective states that our bodies constitute a starting point from which we perceive the world, and that we continuously reshape all our experiences through our bodies. Consequently, we both are our diseases as well as having them. From this point of view, disease and physical and/or psychological changes are continuously integrated in our bodies and minds since we feel, think and act as a whole.

Also, we understand ourselves when a possibility to interpret our feelings, thoughts and actions as a whole, is given to us. Therefore, understanding and interpretation create life quality, self-esteem and self-solicitude.

In this understanding of ourselves, there must exist a mutual relationship between humans, where respect for the human always precedes respect of the norms. This relationship allows a person's entire existence to enter into a dialogue with things that maybe exist, which in turn opens up possibilities for a fictive world of possible events. These possible fictive events can then pave the way for change.

\subsubsection{Caring Science - Person-centered care}

Caring science incorporates arts and humanities as well as science $[33,34]$. Inspired by the phenomenological lifeworld perspective [31,32] caring scientists like Watson [35] and Parse [36] and others representing person-centered care, it is argued that our understanding of ourselves is based on mutual relationship between humans [37-39]. Consequently, caring can only be demonstrated and practiced interpersonally-in the context of a relationship that is established between the caregiver and the person being cared for. In this context, the person in care is regarded as a capable human being ("homo capax") in relation to the care and the caregiver. The relationship between the caregiver and the person being cared for is always in clinical focus or, "paramount", as McCormack and McCance [39] term it.

\subsubsection{Music Therapy}

The American Music Therapy Association defines music therapy as "the clinical and evidence-based use of music interventions to accomplish individualized goals within a therapeutic relationship by a credential professional who has completed an approved therapy program" [40]. The World Federation of Music Therapy [41] presents another definition of music therapy as the "professional use of music and its elements as an intervention..." . The variation in definitions can be

1"Music therapy is the professional use of music and its elements as an intervention in medical, education, and everyday environments with 
derived from the fact that music therapy contains many different perspectives, approaches, models, techniques and methods. For example, Bruscia [42] describes four main methods: improvising, recreating (or performing), composing and listening to music, each with its own therapeutic potential and applications. Edwards [43] uses concept-like approaches and models for describing the field, such as the Nordoff Robbins music therapy model, Community music therapy and others. The music therapy used in PCMusic is influenced by perspectives from the music therapy field viz. humanistic [44], psychodynamic [45], aesthetic [46], resource-oriented [47] and evidencebased practice music therapy [48]. This mix of perspectives fits well in the caring and medical environments expressing respect for the processes in a patient's body, mind and spirit in these complex settings and environments.

\subsubsection{Integrated Mental Training}

Patients use of music as a self-management technique (self-coaching) to reduce or control distress has been explored and described in nursing [49]. A broader approach of self-management for increased personal development and higher quality of life is presented in Integrated Mental Training (IMT) by Uneståhl[50]. IMT consists of listening to study material in an alternative state of consciousness where an inner mental room is used as an operational space. However, in PCMusic listening to music constitutes the main activity compared to listening to IMT's study materials. The patient together with the music therapist, creates PCMusic, a list of self-selected music appropriate for personalized treatment. The patient then repeatedly listens to the list of music in a relaxed state of mind, guided in an emotional positive way by the music therapist. Then, after some time with repeated pairing, the music will arouse positive emotions even in the absence of the music therapist. The act of pairing pieces of music and positive stimuli are in PCMusic thus used as "evaluated conditioning", a process described by Juslin[7, 51] where an emotion is induced by a piece of music because the music repeatedly has been "associated" with a positive stimulus. Conditioning enables shaping the emotional responses to music in a subtle way due to the involvement of subconscious, unintentional, and effortless processes [7].

\subsection{Procedure}

The method PCMusic contains five phases, each with one or more steps or actions forming a procedure, which is defined as an organized sequence of operations and interactions. The phases and steps should be regarded as

individuals, groups, families, or communities who seek to optimize their quality of life and improve their physical, social, communicative, emotional, intellectual, and spiritual health and wellbeing. Research, practice, education, and clinical training in music therapy are based on professional standards according to cultural, social, and political contexts" [45]. suggestions since PCMusic should be both flexible and adjusted to the actual patient within the specific context. The steps should also consider continuous evaluation of the patient and context.

\subsubsection{Phase 1 Start-up}

The start-up depends on the patient and context, and involves implementing the method and procedures as a collaboration between the music therapist and the healthcare professionals. The healthcare professionals and the therapist discuss the proposed interventions from a pain- and treatment perspective so that the caregivers are informed about how the intervening procedure is administered both physically and temporally.

\subsubsection{Phase 2 Music Therapy}

Step 1. After start-up, the patient is invited to have one or more individual sessions with the music therapist. Together they explore the patient's musical preferences and narratives (can be complemented by a written narrative). With the patient's agreement, the music therapist then shares relevant information with healthcare professionals and a dialogue between patient and staff is offered. Through this collaboration, both patient and caregiver can gain an understanding of the patient's experiences, which, together with pathology, can give the healthcare professionals a basis for discussing and planning individualized care and treatment with the patient. The collaboration also establishes a provider-patient partnership between the caregiver and the patient which encourages and empowers the patient to actively take part in finding solutions to possible problems.

Step 2. During this step, the music therapist creates some media containing the self-selected music. The style, type or specific music pieces that are chosen depends the patient's preferences and narratives as well as music relevant from a music therapeutic perspective. For example, if the intervention contains a peak in pain, the therapist creates the media with that in mind.

Step 3. This step consists of additional music therapy sessions. During these sessions, the patient performs relaxation exercises while listening to the music, without any medical treatment being given. The music therapist here guides the patient to reach an alternative state of consciousness where an "inner mental room" is created and this room is used as an operational space. In this inner room, the mental processes that affect the body can be more easily reached.

The patient is offered relaxation exercises while listening to music with the objective of associate the positive experiences of the exercises with music. After the patient has achieved the desired level of relaxation and is satisfied with his or her inner mental room, the music media used is handed to the patient for further training without the presence of the music therapist. 


\subsubsection{Phase 3 Self-coaching}

In this phase the patient continues to practice the relaxation exercises while listening to the preferred music without the music therapist. When the patient controls relaxation by listening to the chosen music, the patient is then ready to use the music during treatment (or other situations of pain).

The patient can need additional music therapy sessions, which can be given until the patient can control the experience satisfactorily so that positive emotions and wellbeing through relaxation during music listening is achieved.

\subsubsection{Phase 4 Caring intervention and/or medical treatment}

The patient now uses self-coaching and music listening while being subjected to painful caring intervention and/or medical treatment. During the intervention, the patient manages the music player and decides whether to use headphones or loudspeakers. Since the music therapist does not participate in this phase it requires good collaboration with the healthcare staff. For example, the staff must be aware of, and manage, the fact that different kind of music may be heard within the treatment room (if loud speakers are used), and that caregivers must communicate with the patient in such a way that it does not disturb the patient's alternate state of consciousness.

\subsubsection{Phase 5 Evaluation}

Since PCMusic and other methods used as complementary methods for pain control only have a small body of evidence, method evaluation is important.

The evaluation should consider the setting as well as context. We recommend that both quantitative and qualitative evaluations are used.

\section{Discussion}

PCMusic is here discussed from the phenomenological life-world perspective followed by a discussion of the music therapist as a provider in care. The question of selfcoaching and the use of preferred or self-selected music in pain management is also addressed.

\subsection{Self-solicitude}

Patient's perception of pain and quality of life are affected by the fact that they experience the world through the phenomenon of pain. According to Ricœur [32], life quality can only be achieved through having the possibility to interpret and understand oneself as well as one's actions. Interpretation and understanding thereby have a relationship. If a person is given the possibility to explore and evaluate himself, self-solicitude can grow out of selfesteem and self-solicitude is regarded as especially important in mitigating suffering. This means that it is important to give a patient the possibility to develop enough self-esteem, so he can achieve self-solicitude and consequentially gain a higher quality of life, despite suffering pain. One way of increasing solicitude is to facilitate healthy coping strategies and a sense of control/empowerment [29]. A similar reasoning is used for PCMusic, in where patient flexibility is stressed. The possibility to adjust to what each patient needs in each specific situation and context to achieve a better life quality is hereby increased.

\subsection{The Music Therapist as a Provider in Care}

Can PCMusic be referred to as music therapy, or, music used in person-centered care? Considering the definition of music therapy presented by WFMT (2011) one could argue that PCMusic belongs to the field of music therapy. On the other hand, The American Music Therapy Associations' definition of music therapy suggests that only a person who has been trained in music therapy can perform music therapy, which raises the question of the role of the music therapist in PCMusic.

PCMusic requires cooperation between healthcare professionals and a music therapist since it would otherwise be difficult for a patient to receive enough pain reduction with music during caring/medical treatment, and healthcare professionals would have difficulties knowing how to meet a patient's musical needs during treatment. Loewy [28], states that music therapists should be included in therapeutic teams for best results. In Ghetti's[29] enquiry, the role of the music therapist is also highlighted as one of main provider and validator.

As a method, the procedures in PCMusic show similarities with the model presented by Ghetti [29] —A procedure of phases with steps describing how to prepare the patient-, while PCMusic involves engaging both therapist and healthcare staff in preparing the work together. Ghetti also proposes patient engagement, a proposition that is of central interest in PCMusic as it involves self-coaching or self-management. Ghetti also emphasizes the importance of on-going assessment and the reflexivity that a music therapist continually performs. In PCMusic, assessments and reflexive procedures are performed both by the music therapist as well as caregivers. The closest similarity, and also the most significant and common trait between the present method and Ghetti's, is the individualized approach, i.e. to enable continuous modification of the treatment according to the patient's needs and responses. This individualistic approach corresponds well with the phenomenological life-world perspective and person-centered care. In conclusion, PCMusic should be referred to as person-centered music, and not music used in person-centered care or music therapy. PCMusic is based on a collaboration among a patient, healthcare professionals and a music therapist, and not only the relationship between the patient and the music 
therapist.

\subsection{Self-coaching Music}

Emotions can induce health outcomes, be an indicator of health as well as motivate health-promoting behaviors [10, 52], and listening to music can arouse profound emotions in humans [7].

Melzack[3] describes how the cortex can modify and prevent pain signals by affecting and controlling inhibitory messages and that one input factor in the pain processes is motivational-affective, i.e. emotions. This is contradicted by Roy et al. [20] who states that music evokes emotional responses due to distraction. There exists a correlation between music and positive emotions [11], and it is music that evokes positive emotions that are selected and used in PCMusic. The patient is listening to music based on the patients' own musical preferences and musical narratives. Through relaxation exercises combined with listening to the chosen music, they create an "inner mental room" which is "charged", conditioned, with positive emotions and thoughts that help the patient to handle and overcome pain. This "self-coaching" music can be brought everywhere to counteract pain.

\subsection{Preferred or self-selected Music}

The question of preferred music or self-selected music for pain management is ambiguous. Listening to preferred music or self-selected music both partially correlates with feelings of control, memories and associations which in turn can promote a sense of control over pain [53], where self-selected music seems to compare favorably $[14,21]$. Some researchers propose portable self-selected music listening for pain relief since it is a low-cost treatment available for many patients with pain [30]. The self-selected music chosen by patients and music therapists in collaboration, as in PCMusic, contain both the benefits that self-selected music gives as well as the musical knowledge provided by the music therapist.

\section{Concluding Remarks}

The ambition to create an interdisciplinary method involving music as a complementary treatment for pain management which improves patient's health and wellbeing, has resulted in a more multidimensional method than Ghetti's proposed model.

One difficulty with PCMusic is the allocation of additional time as the implementation in a caring/medical environment requires collaboration among patient, music therapist and healthcare professionals. Other difficulties of PCMusic are; possible elevation of caregiver stress due to added complexity, different musical preferences and possible ambient noise, and the need for increased financial resources.
Despite the current lack of evidence, we believe that it is possible to both evaluate PCMusic and support patients with pain for greater health and wellbeing. PCMusic needs to be tested in several trials and/or pilot studies to determine its' efficacy. To evaluate the method, we recommend both quantitative and qualitative evaluations since humans, pain, music, settings and context are complex areas. For example, Hanser[8] believes that it is "immensely" problematic to control all the variables that could affect the perception of pain using music, and therefore propose self-reports as the most valuable pain assessment method.

We believe that it is important to describe and test different kinds of working models or methods within the area of evidenced-based practice. Our opinion is also that the subject field of music used as a complementary treatment in pain management needs more studies with higher ecological validity.

If evidence is shown for the efficacy of PCMusic, patients with pain can be offered with a cost-effective complementary treatment without side effects. Since treatment with PCMusic can be brought into different kinds of unfamiliar settings and environments; clinics, hospitals and care centers, it also can be offered to patients who suffer from other conditions than pain e.g. diseases and treatments connected to stress, fear and anxiety. If the results of PCMusic show positive experiences of emotions during music listening relieving pain, it opens up for the possibility to develop a wide range of methods and courses of action that can be employed in healthcare and medicine to support patients in different kind of areas. A consequence is that specific knowledge about (how to influence) emotions could be used in care and medical settings as well as in educational environments.

\section{Acknowledgements}

This work was supported by Sophiahemmet Foundation, Stockholm, Sweden. Special thanks to professor Töres Theorell for his invaluable medical expertise and to associated professor Ralf Torgrip for linguistic revision.

\section{REFERENCES}

[1] A. Olofsson, B. Fossum, Perspectives on music therapy in adult cancer care: a hermeneutic study. Oncol Nurs Forum, Vol. 36, No. 4, 223-31, 2009.

[2] IASP, IASP Terminology, H. Merskey and N. Bogduk, Editors., IASP Press: Seattle, 2017.

[3] R. Melzack, Pain and the neuromatrix in the brain. Journal of dental education, Vol. 65, No. 12, 1378-1382, 2001.

[4] H. Breivik, et al., Survey of chronic pain in Europe: 
prevalence, impact on daily life, and treatment. European journal of pain, Vol. 10, No. 4, 287-287, 2006.

[5] C. Phillips, et al., Prioritising pain in policy making: The need for a whole systems perspective. Health Policy, Vol. 88, No. 2, 166-175, 2008.

[6] D. Lundberg, S. Axelsson, A systematic review of the scientific literature on the treatment of chronic pain. Läkartidningen, 2006.

[7] P. N. Juslin, From everyday emotions to aesthetic emotions: Towards a unified theory of musical emotions. Physics of Life Reviews, Vol. 10, No. 3, 235-266, 2013.

[8] S. Hanser, The Role of Music in Pain Management, in Handbook of Music and Emotion Theory, Research, Applications, P.N. Juslin and J.A. Sloboda, Editors., Oxford University Press: Oxford, 2010.

[9] U. Nilsson, The anxiety- and pain-reducing effects of music interventions: a systematic review. Aorn j, Vol. 87, No. 4, 780-807, 2008.

[10] H. Leventhal, L. Patrick-Miller, Emotions and physical illness: Causes and indicators of vulnerability, in Handbook of emotions, M. Lewis and J.M. Haviland-Jones, Editors., Guilford Press: New York. 645-60, 2000.

[11] D. Västfjäll, P. Juslin, T. Hartig, Music, Subjective Wellbeing, and Health: The Role of Everyday Emotions, in Music, Health, \& Wellbeing, R. A. R. MacDonald, G. Kreutz, and L. Mitchell, Editors., Oxford University Press: Oxford, 2012.

[12] D. Knox, et al., Acoustic analysis and mood classification of pain-relieving music. J Acoust Soc Am, Vol. 130, No. 3, 1673-82, 2011.

[13] C. J. Brown, A. C. N. Chen, S. F. Dworkin, Music in the Control of Human Pain. Music Therapy, Vol. 8, No. 1, 47-60, 1989.

[14] R. Spintge, Thirty-five Years Use of Anxiolytic Music (AAM) in Pain and Aversive Clinical Settings, in Music and Medicine. Integrative Methods in the Treatment of Pain, J. F. Mondanaro and G. A. Sara, Editors., NY: Satchnote Press: New York, 2013.

[15] A. C. North, D. J. Hargreaves, Lifestyle correlates of musical preference: 1. Relationships, living arrangements, beliefs, and crime. Psychology of Music, Vol. 35, No. 1, 58-87, 2007.

[16] J. A. Sloboda, P. N. Juslin, Psychological perspectives on music and emotion, in Music and Emotion, P. N. Juslin and J. A. Sloboda, Editors., Oxford University Press: Oxford, 2001.

[17] H. M. Hekmat, J. B. Hertel, Pain Attenuating Effects of Preferred Versus Non-preferred Music Interventions. Psychology of Music, Vol. 21, No. 2, 163-173, 1993.

[18] L. Mitchell, R. A. R. MacDonald, An experimental investigation of the effects of preferred and relaxing music listening on pain perception. J Music Ther, Vol. 43, No. 4, 295-316, 2006.

[19] D. E. deGood, Relationship of pain coping strategies to adjustment and functioning, in Personality Characteristics of Patients with Pain, R. J. Gatchel and J. N. Wesiberg, Editors.,
American Pain Society: Washington, D C. 129-64, 2000.

[20] M. Roy, I. Peretz, P. Rainville, Emotional valence contributes to music-induced analgesia. Pain, Vol. 134, No. $1-2,140-7,2008$

[21] H. Zhao, A. C. Chen, Both happy and sad melodies modulate tonic human heat pain. J Pain, Vol. 10, No. 9, 953-60, 2009.

[22] L. Mitchell, R. A. R. MacDonald, Music and Pain: Evidence from Experimental Perspectives, in Music, Health, \& Wellbeing, R. A. R. MacDonald, G. Kreutz, and L. Mitchell, Editors., Oxford University Press: Oxford, 2012.

[23] J. Lingham, T. Theorell, Self-selected "favourite" stimulative and sedative music listening - how does familiar and preferred music listening affect the body? Nordic Journal of Music Therapy, Vol. 18, No. 2, 150-166, 2009.

[24] A. Linnemann, et al., The effects of music listening on pain and stress in the daily life of patients with fibromyalgia syndrome. Frontiers in Human Neuroscience, Vol. 9, No., 434,2015

[25] S. Koelsch, T. Stegemann, The Brain and Positive Biological Effects in Healthy and Clinical Populations, in Music, Health \& Wellbeing, R. A. R. MacDonald, G. Mitchell, L. , Editor., Oxford University Press: Oxford, 2012.

[26] T. Masuda, K. Miyamoto, K. Shimizu, Effects of Music Listening on Elderly Orthopaedic Patients During Postoperative Bed Rest. Nordic Journal of Music Therapy, Vol. 14, No. 1, 4-14, 2005.

[27] A. Ullsten, et al., Family-centred music therapy during painful procedures in neonatal care, in PEARL/PICH2Gomeeting. Copenhagen, 2018.

[28] J. Loewy, Music Sedation and Pain, in Music and Medicine. Integrative Methods in the Treatment of Pain, J. F. Mondanaro and G. A. Sara, Editors., NY: Satchnote Press: New York, 2013.

[29] C. M. Ghetti, Music therapy as procedural support for invasive medical procedures: toward the development of music therapy theory. Nordic Journal of Music Therapy, Vol. 21, No. 1, 3-35, 2012.

[30] D. J. Hargreaves, R. A. R. MacDonald, D. Miell, Musical Imaginations, in Musical Imaginations, D.J. Hargreaves, MacDonald, R. A. R., \& Miell, D. , Editor., Oxford University Press: Oxford, 2012.

[31] M. Merleau-Ponty, Phenomenology of Perception. New York: Routledge \& Kegan Paul, 2005.

[32] P. Ricœur, Oneself as another. Chicago: Univ. of Chicago Press, 1992.

[33] J. Fawcett, et al., On Nursing Theories and Evidence. Journal of Nursing Scholarship, Vol. 33, No. 2, 115-119, 2001.

[34] A. I. Meleis, Theoretical nursing: Development and progress: USA: Lippincott Williams \& Wilkins, 2011.

[35] J. Watson, Nursing: the philosophy and science of caring. Boulder: University Press of Colorado, 2008.

[36] R. R. Parse, The human becoming school of thought. California: Thousand Oaks, 1998.

[37] I. Ekman, et al., Person-centered care--ready for prime time. 
Eur J Cardiovasc Nurs, Vol. 10, No. 4, 248-51, 2011.

[38] B. McCormack, J. Dewing, T. McCance, Developing person-centered care: addressing contextual challenges through practice development. OJIN, Vol. 16, No. 2, 2011.

[39] B. McCormack, T. McCance, Person-centred practice in nursing and healht care: theory and practice. Chichester: Wiley-Blackwell, 2017.

[40] The American Music Therapy Association. 2018.

[41] The World Federation of Music Therapy. 2011.

[42] K. Bruscia, Defining music therapy. USA: Barcelona Publishers, 2014.

[43] J. Edwards, Conceptualizing Music Therapy, in The Oxford Handbook of Music Therapy, J. Edwards, Ed., Oxford University Press: Oxford, 2016.

[44] L. Bunt, B. Stige, Music therapy: An art beyond words. Routledge, 2014.

[45] S. Metzner, Psychodynamic Music Therapy, in The Oxford Handbook of Music Therapy, J. Edwards., Ed., Oxford University Press: Oxford, 2016.

[46] C. A. Lee, Aesthetic Music Therapy, in The Handbook of Music Therapy, J. Edwards, Ed., Oxford University Press: Oxford, 2016.
[47] R. Rolvsjord, Five episodes of clients' contributions to the therapeutic relationship: a qualitative study in adult mental health care. Nordic Journal of Music Therapy, Vol. 25, No. 2, 159-184, 2016.

[48] F. A. Baker, Evidence-Based Practice in Music Therapy, in Music Therapy Handbook, B. Wheeler, Ed., The Guilford Press: New York and London, 2015.

[49] U. Nilsson, The Effect of Music and Music in Combination With Therapeutic Suggestions on Postopreative Recovery. Linköping University: Linköping, 2003.

[50] L.-E. Uneståhl, Integrated Mental Training. Sweden: Mental Training AB, 2011

[51] P. N. Juslin, Musical emotions explained. Oxford: Oxford University Press, 2019.

[52] M. A. Diefenbach, et al., Affect and screening for cancer: A self-regulation perspective, in Handbook of emotions, M. Lewis, J.M. Haviland-Jones, and L.F. Barrett, Editors., Guilford Press: New York. 645-60, 2008.

[53] P. Janata, S. T. Tomic, S. K. Rakowski, Characterisation of music-evoked autobiographical memories. Memory, Vol. 15, No. 8, 845-860, 2007. 